\title{
SENSITIVITY ANALYSIS FOR RELAXED COCOERCIVE NONLINEAR QUASIVARIATIONAL INCLUSIONS
}

\author{
RAM U. VERMA
}

Received 29 June 2005; Revised 2 August 2005; Accepted 5 August 2005

Some results on the sensitivity analysis for relaxed cocoercive quasivariational inclusions are obtained, which generalize similar sensitivity analysis results on strongly monotone quasivariational inclusions. Furthermore, some suitable examples of relaxed cocoercive mappings are illustrated.

Copyright (C) 2006 Ram U. Verma. This is an open access article distributed under the Creative Commons Attribution License, which permits unrestricted use, distribution, and reproduction in any medium, provided the original work is properly cited.

\section{Introduction and preliminaries}

Variational inequality methods whether based on numerous available new algorithms or otherwise have been applied vigorously, especially to model equilibria problems in economics, optimization and control theory, operations research, transportation network modeling, and mathematical programming, while a considerable progress to developing general methods for the sensitivity analysis for variational inequalities is made. Tobin [7] presented the sensitivity analysis for variational inequalities allowing the calculation of derivatives of solution variables with respect to perturbation parameters, where perturbations are of both the variational inequality function and the feasible region. Kyparisis [5] under appropriate second-order and regularity conditions has shown that the perturbed solution to a parametric variational inequality problem is continuous and directionally differentiable with respect to the perturbation parameter. Recently, Agarwal et al. [1] studied the sensitivity analysis for qusivariational inclusions involving strongly monotone mappings applying the resolvent operator technique, without differentiability assumptions on solution variables with respect to perturbation parameters. The aim of this paper is to present the sensitivity analysis for the relaxed cocoercive quasivariational inclusions based on the resolvent operator technique. The obtained results generalize the results on the sensitivity analysis for strongly monotone quasivariational inclusions $[1,2,6]$ and others since the class of relaxed cocoercive mappings is more general than the strong monotone mappings, and furthermore, it is less explored. Some suitable

Hindawi Publishing Corporation

Journal of Applied Mathematics and Stochastic Analysis

Volume 2006, Article ID 52041, Pages 1-9

DOI 10.1155/JAMSA/2006/52041 
examples of relaxed cocoercive mappings are also included. For more details, we recommend [1-13].

Let $H$ be a real Hilbert space with the norm $\|\cdot\|$ and inner product $\langle\cdot, \cdot\rangle$. Let $N: H \times$ $H \times L \rightarrow H$ be a nonlinear mapping and $M: H \times H \times L \rightarrow 2^{H}$ be a maximal monotone mapping with respect to the first variable, where $L$ is a nonempty open subset of $H$. Then the problem of finding an element $u \in H$ such that

$$
0 \in N(u, u, \lambda)+M(u, u, \lambda)
$$

where $\lambda \in L$ is the perturbation parameter, is called a class of generalized relaxed cocoercive mixed quasivariational inclusion (abbreviated RCMQVI) problems.

Next, a special case of RCMQVI (1.1) problem is: determine an element $u \in H$ such that

$$
0 \in S(u, \lambda)+T(u, \lambda)+M(u, u, \lambda)
$$

where $N(u, v, \lambda)=S(u, \lambda)+T(v, \lambda)$, for all $u, v \in H$, and $S, T: H \times L \rightarrow H$ are two nonlinear mappings. If $S=0$ in (1.2), then (1.2) is equivalent to finding an element $u \in H$ such that

$$
0 \in T(u, \lambda)+M(u, u, \lambda)
$$

The solvability of RCMQVI problem (1.1) depends on the equivalence between (1.1) and the problem of finding the fixed point of the associated resolvent operator.

Note that if $M$ is maximal monotone, then the corresponding resolvent operator $J_{\rho}^{M}$ in the first argument is defined by

$$
J_{\rho}^{M(\cdot, y)}(u)=(I+\rho M(\cdot, y))^{-1}(u) \quad \forall u \in H
$$

where $\rho>0$ and $I$ is the identity mapping.

Lemma 1.1 [1]. An element $u \in H$ is a solution to (1.1) if and only if there is an element $u \in H$ such that

$$
u=G(u, \lambda):=J_{\rho}^{(\cdot, u, \lambda)}(u-\rho N(u, u, \lambda))
$$

where $J_{\rho}^{(\cdot, u, \lambda)}=(I+\rho M(\cdot, u, \lambda))^{-1}$ and $\rho>0$.

\section{Cocoercivity and relaxed cocoercivity}

This section deals with notions of cocoercive and relaxed cocoercive mappings and their connections to other mappings. The class of relaxed cocoercive mappings is more general than the strong monotone mappings, and furthermore, it is less explored in the context of applications yet. 
Definition 2.1. A mapping $T: H \times H \times L \rightarrow H$ is said to be

(i) $(m)$-relaxed monotone in the first argument if there exists a positive constant $m$ such that

$$
\langle T(x, u, \lambda)-T(y, u, \lambda), x-y\rangle \geq(-m)\|x-y\|^{2} \quad \forall(x, y, u, \lambda) \in H \times H \times H \times L ;
$$

(ii) (s)-cocoercive in the first argument if there exists a positive constant $s$ such that

$$
\langle T(x, u, \lambda)-T(y, u, \lambda), x-y\rangle \geq(s)\|T(x)-T(y)\|^{2} \quad \forall(x, y, u, \lambda) \in H \times H \times H \times L ;
$$

(iii) $(m)$-relaxed cocoercive in the first argument if there exists a positive constant $m$ such that

$$
\langle T(x, u, \lambda)-T(y, u, \lambda), x-y\rangle \geq(-m)\|T(x)-T(y)\|^{2} \quad \forall(x, y, u, \lambda) \in H \times H \times H \times A L ;
$$

(iv) $(\gamma, m)$-relaxed cocoercive in the first argument if there exist positive constants $\gamma$ and $m$ such that

$$
\begin{aligned}
& \langle T(x, u, \lambda)-T(y, u, \lambda), x-y\rangle \\
& \quad \geq(-m)\|T(x)-T(y)\|^{2}+\gamma\|x-y\|^{2} \quad \forall(x, y, u, \lambda) \in H \times H \times H \times L .
\end{aligned}
$$

Example 2.2. Consider a nonexpansive mapping $T: H \rightarrow H$ on $H$. If we set $A=I-T$, then $A$ is (1/2)-cocoercive.

Example 2.3. Consider a projection $P: H \rightarrow C$, where $C$ is a nonempty closed convex subset of $H$. Then $P$ is (1)-cocoercive since $P$ is nonexpansive.

Example 2.4. Consider an $(r)$-strongly monotone (and hence $(r)$-expanding) mapping $T: H \rightarrow H$ on $H$. Then $T$ is $\left(1, r+r^{2}\right)$-relaxed cocoercive. For all $u, v \in H$, we have

$$
\begin{aligned}
\langle T(x)-T(y), x-y\rangle & =(-1)\|T(x)-T(y)\|^{2}+r\|x-y\|^{2}+\|T(x)-T(y)\|^{2} \\
& \geq(-1)\|T(x)-T(y)\|^{2}+\left(r+r^{2}\right)\|x-y\|^{2} .
\end{aligned}
$$

Clearly, every $(m)$-cocoercive mapping is $(m)$-relaxed cocoercive, while each $(r)$-strongly monotone mapping is $\left(1, r+r^{2}\right)$-relaxed cocoercive.

Definition 2.5. A mapping $T: H \times H \times L \rightarrow H$ is said to be $(\mu)$-Lipschitz continuous in the first argument if there exists a positive constant $\mu$ such that

$$
\|T(x, u, \lambda)-T(y, u, \lambda)\| \leq \mu\|x-y\| \quad \forall(x, y, u, \lambda) \in H \times H \times H \times L .
$$


4 Sensitivity analysis

\section{Nonlinear variational inclusions}

Theorem 3.1. Let $H$ be a real Hilbert space, let $N: H \times H \times L \rightarrow H$ be $(\gamma, r)$-relaxed cocoercive and $(\beta)$-Lipschitz continuous in the first variable, and let $N$ be $(\mu)$-Lipschitz continuous in the second variable. If

$$
\left\|J_{\rho}^{M(\cdot, u, \lambda)}(w)-J_{\rho}^{M(\cdot, v, \lambda)}(w)\right\| \leq \eta\|u-v\| \quad \forall(u, v, \lambda) \in H \times H \times L,
$$

then

$$
\|G(u, \lambda)-G(v, \lambda)\| \leq \theta\|u-v\| \quad \forall(u, v, \lambda) \in H \times H \times L
$$

where

$$
\begin{gathered}
\theta=\sqrt{1-2 \rho r+\rho^{2} \beta^{2}+2 \rho \gamma \beta^{2}}+\rho \mu+\eta<1 \\
\left|\rho-\frac{r-(1-\eta) \mu-\gamma \beta^{2}}{\beta^{2}-\mu^{2}}\right|<\frac{\sqrt{\left(r-(1-\eta) \mu-\gamma \beta^{2}\right)^{2}-\left(\beta^{2}-\mu^{2}\right)(2-\eta) \eta}}{\beta^{2}-\mu^{2}} \\
r>(1-\eta) \mu+\gamma \beta^{2}+\sqrt{\left(\beta^{2}-\mu^{2}\right)(2-\eta) \eta}, \quad \beta>\mu \\
\rho \mu<1-\eta, \eta<1 .
\end{gathered}
$$

Consequently, for each $\lambda \in L$, the mapping $G(u, \lambda)$ in light of (3.2) has a unique fixed point $z(\lambda)$, and hence, $z(\lambda)$ is a unique solution to (1.1). Thus,

$$
G(z(\lambda), \lambda)=z(\lambda)
$$

Proof. For any element $(u, v, \lambda) \in H \times H \times L$, we have

$$
\begin{aligned}
& G(u, \lambda)=J_{\rho}^{M(\cdot, u, \lambda)}(u-\rho N(u, u, \lambda)), \\
& G(v, \lambda)=J_{\rho}^{M(\cdot, v, \lambda)}(v-\rho N(v, v, \lambda)) .
\end{aligned}
$$

It follows that

$$
\begin{aligned}
&\|G(u, \lambda)-G(v, \lambda)\| \\
&=\left\|J_{\rho}^{M(\cdot, u, \lambda)}(u-\rho N(u, u, \lambda))-J_{\rho}^{M(\cdot, v, \lambda)}(v-\rho N(v, v, \lambda))\right\| \\
& \leq\left\|J_{\rho}^{M(\cdot, u, \lambda)}(u-\rho N(u, u, \lambda))-J_{\rho}^{M(\cdot, u, \lambda)}(v-\rho N(v, v, \lambda))\right\| \\
&+\left\|J_{\rho}^{M(\cdot, u, \lambda)}(v-\rho N(v, v, \lambda))-J_{\rho}^{M(\cdot, v, \lambda)}(v-\rho N(v, v, \lambda))\right\| \\
& \leq\|u-v-\rho(N(u, u, \lambda)-N(v, v, \lambda))\|+\eta\|u-v\| \\
& \leq\|u-v-\rho(N(u, u, \lambda)-N(v, u, \lambda))\| \\
&+\|\rho(N(v, u, \lambda)-N(v, v, \lambda))\|+\eta\|u-v\| .
\end{aligned}
$$


The $(\gamma, r)$-relaxed cocoercivity and $(\beta)$-Lipschitz continuity of $N$ in the first argument imply that

$$
\begin{aligned}
\| u-v & -\rho(N(u, u, \lambda)-N(v, u, \lambda)) \|^{2} \\
& =\|u-v\|^{2}-2 \rho\langle N(u, u, \lambda)-N(v, u, \lambda), u-v\rangle+\rho^{2}\|N(u, u, \lambda)-N(v, u, \lambda)\|^{2} \\
& \leq\left(1-2 \rho r+\rho^{2} \beta^{2}+2 \rho \gamma \beta^{2}\right)\|u-v\|^{2} .
\end{aligned}
$$

On the other hand, the $(\mu)$-Lipschitz continuity of $N$ in the second argument results in

$$
\|N(v, u, \lambda)-N(v, v, \lambda)\| \leq \mu\|u-v\| .
$$

In light of the above arguments, we infer

$$
\|G(u, \lambda)-G(v, \lambda)\| \leq \theta\|u-v\|
$$

where

$$
\theta=\sqrt{1-2 \rho r+\rho^{2} \beta^{2}+2 \rho \gamma \beta^{2}}+\rho \mu+\eta
$$

Since $\theta<1$, it concludes the proof.

Theorem 3.2. Let $H$ be a real Hilbert space, let $N: H \times H \times L \rightarrow H$ be $(\gamma, r)$-relaxed cocoercive and $(\beta)$-Lipschitz continuous in the first variable, and let $N$ be $(\mu)$-Lipschitz continuous in the second variable. Let

$$
\begin{gathered}
\left\|J_{\rho}^{M(\cdot, u, \lambda)}(w)-J_{\rho}^{M(\cdot, v, \lambda)}(w)\right\| \leq \eta\|u-v\| \quad \forall(u, v, \lambda) \in H \times H \times L, \\
\|G(u, \lambda)-G(v, \lambda)\| \leq \theta\|u-v\| \quad \forall(u, v, \lambda) \in H \times H \times L,
\end{gathered}
$$

where

$$
\begin{gathered}
\theta=\sqrt{1-2 \rho r+\rho^{2} \beta^{2}+2 \rho \gamma \beta^{2}}+\rho \mu+\eta<1, \\
\left|\rho-\frac{r-(1-\eta) \mu-\gamma \beta^{2}}{\beta^{2}-\mu^{2}}\right|<\frac{\sqrt{\left(r-(1-\eta) \mu-\gamma \beta^{2}\right)^{2}-\left(\beta^{2}-\mu^{2}\right)(2-\eta) \eta}}{\beta^{2}-\mu^{2}} \\
r>(1-\eta) \mu+\gamma \beta^{2}+\sqrt{\left(\beta^{2}-\mu^{2}\right)(2-\eta) \eta}, \quad \beta>\mu, \\
\rho \mu<1-\eta, \quad \eta<1 .
\end{gathered}
$$

If the mappings $\lambda \rightarrow N(u, v, \lambda)$ and $\lambda \rightarrow J_{\rho}^{M(\cdot, u, \lambda)}(w)$ are both continuous (or Lipschitz continuous) from $L$ to $H$, then the solution $z(\lambda)$ of (1.1) is continuous (or Lipschitz continuous) from $L$ to $H$. 
Proof. From the hypotheses of the theorem, for any $\lambda, \lambda^{*} \in L$, we have

$$
\begin{aligned}
\left\|z(\lambda)-z\left(\lambda^{*}\right)\right\| & =\left\|G(z(\lambda), \lambda)-G\left(z\left(\lambda^{*}\right), \lambda^{*}\right)\right\| \\
& \leq\left\|G(z(\lambda), \lambda)-G\left(z\left(\lambda^{*}\right), \lambda\right)\right\|+\left\|G\left(z\left(\lambda^{*}\right), \lambda\right)-G\left(z\left(\lambda^{*}\right), \lambda^{*}\right)\right\| \\
& \leq \theta\left\|z(\lambda)-z\left(\lambda^{*}\right)\right\|+\left\|G\left(z\left(\lambda^{*}\right), \lambda\right)-G\left(z\left(\lambda^{*}\right), \lambda^{*}\right)\right\| .
\end{aligned}
$$

It follows that

$$
\begin{aligned}
\left\|G\left(z\left(\lambda^{*}\right), \lambda\right)-G\left(z\left(\lambda^{*}\right), \lambda^{*}\right)\right\|= & \| J_{\rho}^{M\left(\cdot, z\left(\lambda^{*}\right), \lambda\right)}\left(z\left(\lambda^{*}\right)-\rho N\left(z\left(\lambda^{*}\right), z\left(\lambda^{*}\right), \lambda\right)\right) \\
& -J_{\rho}^{M\left(\cdot, z\left(\lambda^{*}\right), \lambda^{*}\right)}\left(z\left(\lambda^{*}\right)-\rho N\left(z\left(\lambda^{*}\right), z\left(\lambda^{*}\right), \lambda^{*}\right)\right) \| \\
\leq & \| J_{\rho}^{M\left(\cdot, z\left(\lambda^{*}\right), \lambda\right)}\left(z\left(\lambda^{*}\right)-\rho N\left(z\left(\lambda^{*}\right), z\left(\lambda^{*}\right), \lambda\right)\right) \\
& -J_{\rho}^{M\left(\cdot, z\left(\lambda^{*}\right), \lambda\right)}\left(z\left(\lambda^{*}\right)-\rho N\left(z\left(\lambda^{*}\right), z\left(\lambda^{*}\right), \lambda^{*}\right)\right) \| \\
& +\| J_{\rho}^{M\left(\cdot, z\left(\lambda^{*}\right), \lambda\right)}\left(z\left(\lambda^{*}\right)-\rho N\left(z\left(\lambda^{*}\right), z\left(\lambda^{*}\right), \lambda^{*}\right)\right) \\
& -J_{\rho}^{M\left(\cdot, z\left(\lambda^{*}\right), \lambda^{*}\right)}\left(z\left(\lambda^{*}\right)-\rho N\left(z\left(\lambda^{*}\right), z\left(\lambda^{*}\right), \lambda^{*}\right)\right) \| \\
\leq & \rho\left\|N\left(z\left(\lambda^{*}\right), z\left(\lambda^{*}\right), \lambda\right)-N\left(z\left(\lambda^{*}\right), z\left(\lambda^{*}\right), \lambda^{*}\right)\right\| \\
& +\| J_{\rho}^{M\left(\cdot, z\left(\lambda^{*}\right), \lambda\right)}\left(z\left(\lambda^{*}\right)-\rho N\left(z\left(\lambda^{*}\right), z\left(\lambda^{*}\right), \lambda^{*}\right)\right) \\
& -J_{\rho}^{M\left(\cdot, z\left(\lambda^{*}\right), \lambda^{*}\right)}\left(z\left(\lambda^{*}\right)-\rho N\left(z\left(\lambda^{*}\right), z\left(\lambda^{*}\right), \lambda^{*}\right)\right) \| .
\end{aligned}
$$

Hence, we have

$$
\begin{aligned}
\left\|z(\lambda)-z\left(\lambda^{*}\right)\right\| \leq & \frac{\rho}{1-\theta}\left\|N\left(z\left(\lambda^{*}\right), z\left(\lambda^{*}\right), \lambda\right)-N\left(z\left(\lambda^{*}\right), z\left(\lambda^{*}\right), \lambda^{*}\right)\right\| \\
& +\frac{1}{1-\theta} \| J_{\rho}^{M\left(\cdot, z\left(\lambda^{*}\right), \lambda\right)}\left(z\left(\lambda^{*}\right)-\rho N\left(z\left(\lambda^{*}\right), z\left(\lambda^{*}\right), \lambda^{*}\right)\right) \\
& -J_{\rho}^{M\left(\cdot, z\left(\lambda^{*}\right), \lambda^{*}\right)}\left(z\left(\lambda^{*}\right)-\rho N\left(z\left(\lambda^{*}\right), z\left(\lambda^{*}\right), \lambda^{*}\right)\right) \| .
\end{aligned}
$$

This completes the proof.

Corollary 3.3 [1]. Let $H$ be a real Hilbert space, let $N: H \times H \times L \rightarrow H$ be $(r)$-strongly monotone and $(\beta)$-Lipschitz continuous in the first variable, and let $N$ be $(\mu)$-Lipschitz continuous in the second variable. If

$$
\left\|J_{\rho}^{M(\cdot, u, \lambda)}(w)-J_{\rho}^{M(\cdot, v, \lambda)}(w)\right\| \leq \eta\|u-v\| \quad \forall(u, v, \lambda) \in H \times H \times L,
$$

then

$$
\|G(u, \lambda)-G(v, \lambda)\| \leq \theta\|u-v\| \quad \forall(u, v, \lambda) \in H \times H \times L,
$$


where

$$
\begin{gathered}
\theta=\sqrt{1-2 \rho r+\rho^{2} \beta^{2}}+\rho \mu+\eta<1, \\
\left|\rho-\frac{r-(1-\eta) \mu}{\beta^{2}-\mu^{2}}\right|<\frac{\sqrt{(r-(1-\eta) \mu)^{2}-\left(\beta^{2}-\mu^{2}\right)(2-\eta) \eta}}{\beta^{2}-\mu^{2}} \\
r>(1-\eta) \mu+\sqrt{\left(\beta^{2}-\mu^{2}\right)(2-\eta) \eta}, \quad \beta>\mu, \\
\rho \mu<1-\eta, \quad \eta<1 .
\end{gathered}
$$

Corollary 3.4 [1]. Let $H$ be a real Hilbert space, let $N: H \times H \times L \rightarrow H$ be $(r)$-strongly monotone and $(\beta)$-Lipschitz continuous in the first variable, and let $N$ be $(\mu)$-Lipschitz continuous in the second variable. Let

$$
\begin{gathered}
\left\|J_{\rho}^{M(\cdot, u, \lambda)}(w)-J_{\rho}^{M(\cdot, v, \lambda)}(w)\right\| \leq \eta\|u-v\| \quad \forall(u, v, \lambda) \in H \times H \times L, \\
\|G(u, \lambda)-G(v, \lambda)\| \leq \theta\|u-v\| \quad \forall(u, v, \lambda) \in H \times H \times L,
\end{gathered}
$$

where

$$
\begin{gathered}
\theta=\sqrt{1-2 \rho r+\rho^{2} \beta^{2}}+\rho \mu+\eta<1, \\
\left|\rho-\frac{r-(1-\eta) \mu}{\beta^{2}-\mu^{2}}\right|<\frac{\sqrt{(r-(1-\eta) \mu)^{2}-\left(\beta^{2}-\mu^{2}\right)(2-\eta) \eta}}{\beta^{2}-\mu^{2}} \\
r>(1-\eta) \mu+\sqrt{\left(\beta^{2}-\mu^{2}\right)(2-\eta) \eta}, \quad \beta>\mu, \\
\rho \mu<1-\eta, \quad \eta<1, \quad r<1 .
\end{gathered}
$$

If the mappings $\lambda \rightarrow N(u, v, \lambda)$ and $\lambda \rightarrow J_{\rho}^{M(\cdot, u, \lambda)}(w)$ are both continuous (or Lipschitz continuous) from $L$ to $H$, then the solution $z(\lambda)$ of (1.1) is continuous (or Lipschitz continuous) from $L$ to $H$.

\section{Concluding remark}

The present results on the sensitivity analysis based on the maximal monotonicity of $M$ can further be generalized to the case of A-monotonicity [9] and H-monotonicity [3]. Recently, the author [9] introduced a new class of mappings - A-monotone mappingswhich have a wide range of applications. The class of $A$-monotone mappings generalizes the well-known class of maximal monotone mappings, and on the other hand, it generalizes the recently introduced and studied notion of the $H$-monotone mappings by Fang and Huang [3] to a higher level.

Let $X$ denote a real Hilbert space with the norm $\|\cdot\|$ and inner product $\langle\cdot, \cdot\rangle$ on $X$.

Definition 4.1 [9]. Let $A: X \rightarrow X$ be a nonlinear single-valued mapping on $X$ and let $M: X \rightarrow 2^{X}$ be a multivalued mapping on $X$. The map $M$ is said to be $A$-monotone if

(i) $M$ is $(m)$-relaxed monotone;

(ii) $A+\rho M$ is maximal monotone for $\rho>0$. 
This is equivalent to stating that $M$ is $A$-monotone if $M$ is $(m)$-relaxed monotone and $\mathrm{R}(A+\rho M)=X$ for $\rho>0$.

Definition 4.2 [3]. Let $H: X \rightarrow X$ be a nonlinear single-valued mapping on $X$ and let $M: X \rightarrow 2^{X}$ be a multivalued mapping on $X$. The map $M$ is said to be $H$-monotone if

(i) $M$ is monotone;

(ii) $(H+\rho M)(X)=X$ for $\rho>0$.

Proposition 4.3. Let $\mathrm{A}: X \rightarrow X$ be an $r$-strongly monotone single-valued mapping and let $M: X \rightarrow 2^{X}$ be an A-monotone mapping. Then $M$ is maximal monotone.

Proof. Since $M$ is $(m)$-relaxed monotone, it suffices to show that

$$
\langle u-v, x-y\rangle \geq(-m)\|x-y\|^{2} \quad \forall(y, v) \in \operatorname{graph}(M) \text { implies } u \in M(x) .
$$

Assume $\left(x_{0}, u_{0}\right) \notin$ graph $(M)$ such that

$$
\left\langle u_{0}-v, x_{0}-y\right\rangle \geq(-m)\left\|x_{0}-y\right\|^{2} \quad \forall(y, v) \in \operatorname{graph}(M) .
$$

Since $M$ is $A$-monotone, $(A+\rho M)(X)=X$ for all $\rho>0$. This implies that there exists an element $\left(x_{1}, u_{1}\right) \in \operatorname{graph}(M)$ such that

$$
A\left(x_{1}\right)+\rho u_{1}=A\left(x_{0}\right)+\rho u_{0} .
$$

It follows from (4.2) and (4.3) that

$$
\rho\left\langle u_{0}-u_{1}, x_{0}-x_{1}\right\rangle=-\left\langle A\left(x_{0}\right)-A\left(x_{1}\right), x_{0}-x_{1}\right\rangle \geq(-\rho m)\left\|x_{0}-x_{1}\right\|^{2} .
$$

Since $A$ is $(r)$-strongly monotone, it implies $x_{0}=x_{1}$ for $m<r$. As a result, we have $u_{0}=u_{1}$, that is, $\left(x_{0}, u_{0}\right) \in$ graph $(M)$, a contradiction. Hence, $M$ is maximal monotone.

Theorem 4.4. Let $A: X \rightarrow X$ be an $r$-strongly monotone mapping and let $M: X \rightarrow 2^{X}$ be an $A$-monotone mapping. Then the operator $(A+\rho M)^{-1}$ is single-valued.

Proof. Assume, for a given $x \in X, u, v \in(A+\rho M)^{-1}(x)$. Then we have $-A(u)+x \in$ $\rho M(u)$ and $-A(v)+x \in \rho M(v)$. Since $M$ is $(m)$-relaxed monotone, it implies that

$$
\begin{aligned}
\langle-A(u)+x-(-A(v)+x), u-v\rangle & =\langle A(v)-A(u), u-v\rangle \\
& \geq(-m)\|u-v\|^{2} .
\end{aligned}
$$

Since $A$ is $(r)$-strongly monotone, it implies $u=v$ for $m<r$. Therefore, $(A+\rho M)^{-1}$ is single-valued.

This leads to the generalized definition of the resolvent operator.

Definition 4.5 [9]. Let A : $X \rightarrow X$ be an ( $r$ )-strongly monotone mapping and let $M: X \rightarrow$ $2^{X}$ be an $A$-monotone mapping. Then the generalized resolvent operator $J_{\rho, A}^{M}: X \rightarrow X$ is defined by

$$
J_{\rho, A}^{M}(u)=(A+\rho M)^{-1}(u)
$$




\section{References}

[1] R. P. Agarwal, Y. J. Cho, and N. J. Huang, Sensitivity analysis for strongly nonlinear quasivariational inclusions, Applied Mathematics Letters 13 (2000), no. 6, 19-24.

[2] X. P. Ding and C. L. Luo, On parametric generalized quasi-variational inequalities, Journal of Optimization Theory and Applications 100 (1999), no. 1, 195-205.

[3] Y. P. Fang and N. J. Huang, $H$-monotone operators and system of variational inclusions, Communications on Applied Nonlinear Analysis 11 (2004), no. 1, 93-101.

[4] H. Iiduka and W. Takahashi, Strong convergence theorem by a hybrid method for nonlinear mappings of nonexpansive and monotone type and applications, Advances in Nonlinear Variational Inequalities 9 (2006), no. 1, 1-9.

[5] J. Kyparisis, Sensitivity analysis framework for variational inequalities, Mathematical Programming 38 (1987), no. 2, 203-213.

[6] A. Moudafi, Mixed equilibrium problems: sensitivity analysis and algorithmic aspect, Computers \& Mathematics with Applications 44 (2002), no. 8-9, 1099-1108.

[7] R. L. Tobin, Sensitivity analysis for variational inequalities, Journal of Optimization Theory and Applications 48 (1986), no. 1, 191-209.

[8] R. U. Verma, Nonlinear variational and constrained hemivariational inequalities involving relaxed operators, Zeitschrift für Angewandte Mathematik und Mechanik 77 (1997), no. 5, 387-391.

[9] _ A-monotonicity and applications to nonlinear variational inclusion problems, Journal of Applied Mathematics \& Stochastic Analysis 2004 (2004), no. 2, 193-195.

[10] - Approximation-solvability of a class of a monotone variational inclusion problems, Journal of Korean Society for Industrial and Applied Mathematics 8 (2004), no. 1, 55-66.

[11]_, Generalized system for relaxed cocoercive variational inequalities and projection methods, Journal of Optimization Theory and Applications 121 (2004), no. 1, 203-210.

[12] W. Y. Yan, Y. P. Fang, and N. J. Huang, A new system of set-valued variational inclusions with $H$-monotone operators, to appear in Mathematical Inequalities and Applications.

[13] E. Zeidler, Nonlinear Functional Analysis and Its Applications. II/B, Springer, New York, 1990.

Ram U. Verma: Department of Theoretical and Applied Mathematics, The University of Akron, Akron, OH 44325-1909, USA

E-mail address: verma99@msn.com 


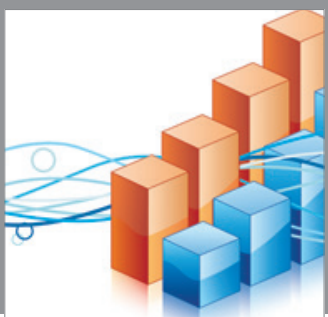

Advances in

Operations Research

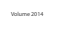

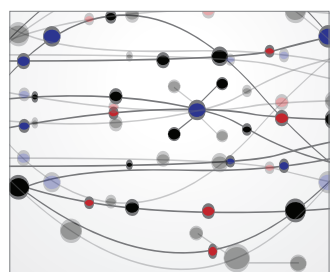

\section{The Scientific} World Journal
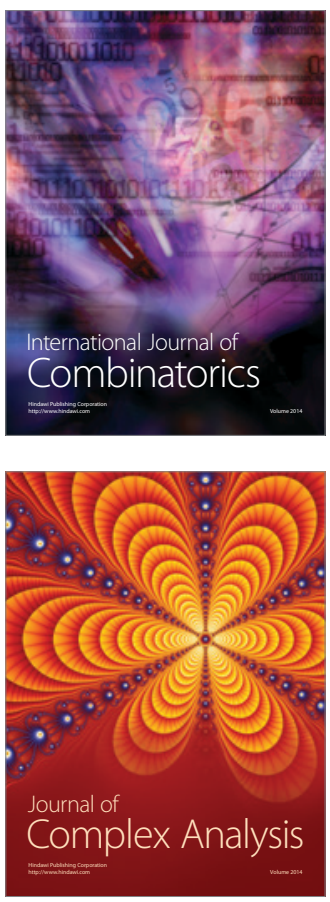

International Journal of

Mathematics and

Mathematical

Sciences
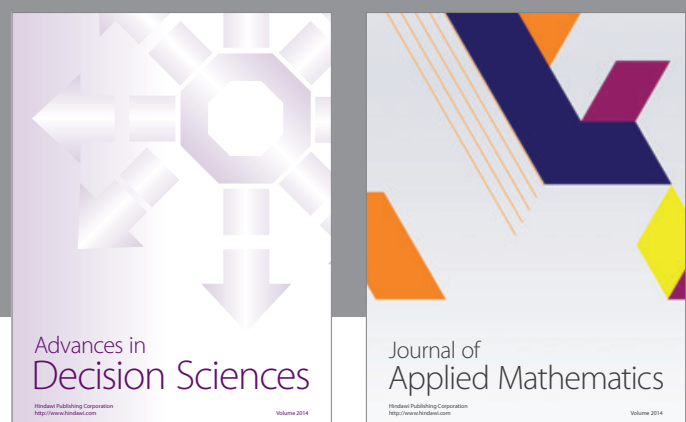

Journal of

Applied Mathematics
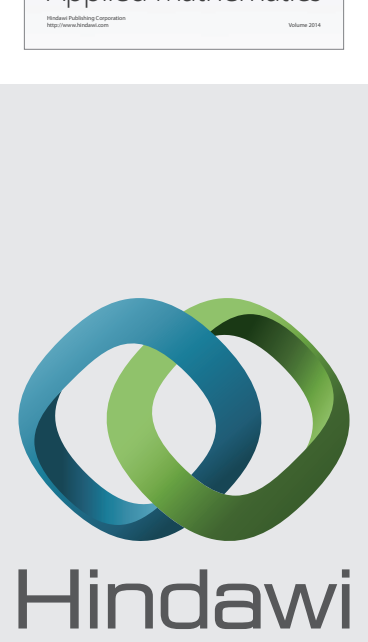

Submit your manuscripts at http://www.hindawi.com
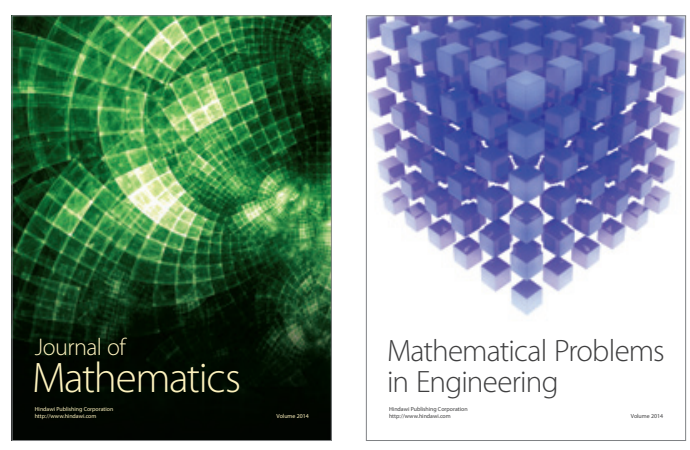

Mathematical Problems in Engineering
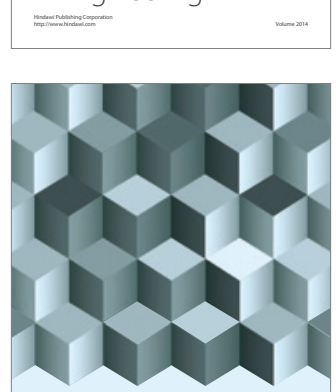

Journal of

Function Spaces
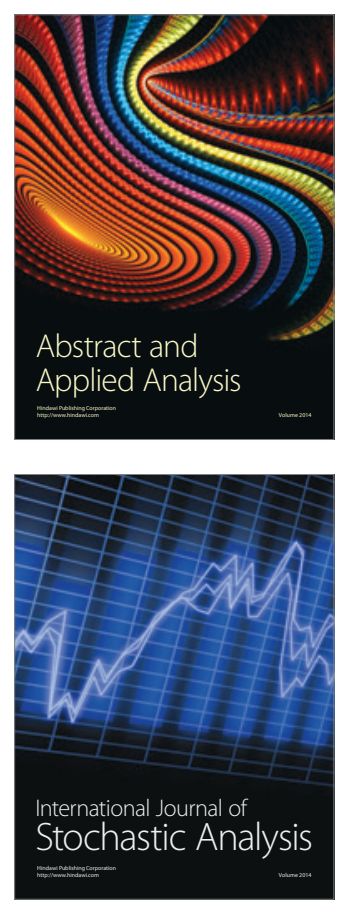

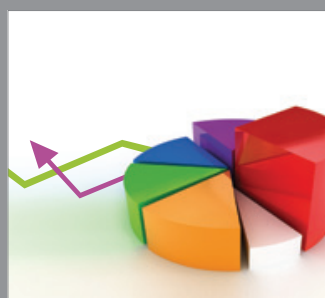

ournal of

Probability and Statistics

Promensencen
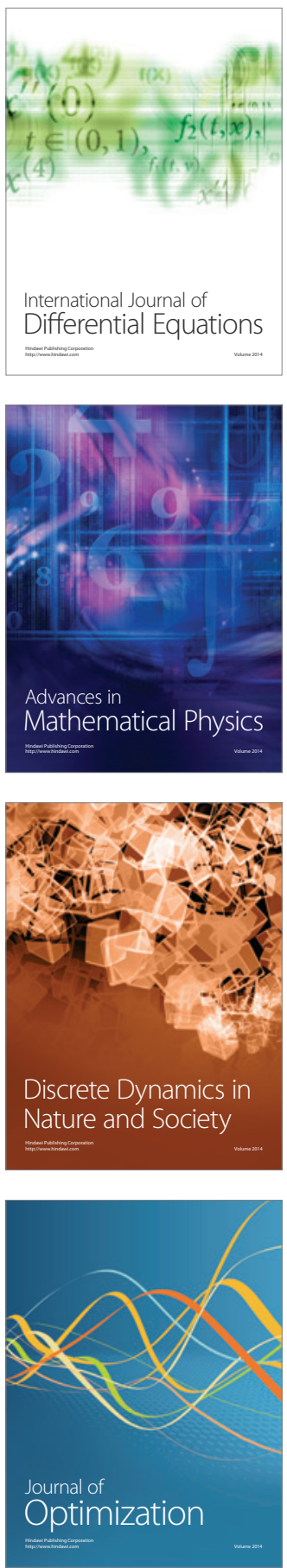